\title{
Black Holes in Gauss-Bonnet Gravity's Rainbow
}

\author{
Seyed Hossein Hendi ${ }^{1,2 *}$ and Mir Faizal ${ }^{3} \dagger$ \\ 1 Physics Department and Biruni Observatory, College of Sciences, Shiraz University, Shiraz 71454, Iran \\ ${ }^{2}$ Research Institute for Astronomy and Astrophysics of Maragha (RIAAM), Maragha, Iran \\ ${ }^{3}$ Department of Physics and Astronomy, University of Waterloo, Waterloo, Ontario, N2L 3G1, Canada
}

(Dated: 29-June-2015)

\begin{abstract}
In this paper, we will generalize the Gauss-Bonnet gravity to an energy dependent Gauss-Bonnet theory of gravity, which we shall call as the Gauss-Bonnet gravity's rainbow. We will also couple this theory to a Maxwell's theory. We will analyze black hole solutions in this energy dependent Gauss-Bonnet gravity's rainbow. We will calculate the modifications to the thermodynamics of black holes in the Gauss-Bonnet's gravity's rainbow. We will demonstrate that even though the thermodynamics of the black holes get modified in the Gauss-Bonnet gravity's rainbow, the first law of thermodynamics still holds for this modified thermodynamics. We will also comment on the thermal stability of the black hole solutions in this theory.
\end{abstract}

\section{INTRODUCTION}

The form of the standard energy-momentum dispersion relation is fixed by the Lorentz symmetry. Even though the Lorentz symmetry is one of the most important symmetries in nature, there are indications from various approaches to quantum gravity that the Lorentz symmetry might be violated in the ultraviolet limit [1] - $[\underline{\underline{5}}$. Thus, it is possible that the Lorentz symmetry is only an effective symmetry which holds in the infrared limit of quantum gravitational processes. As the standard energy-momentum dispersion relation depends on the Lorentz symmetry, it is expected that the standard energy-momentum dispersion relation will also get modified in the ultraviolet limit. In fact, it has been observed that such modification to the standard energy-momentum relation does occur in the discrete spacetime [6], models based on string field theory [7], spacetime foam [8], the spin-network in loop quantum gravity (LQG) [9], non-commutative geometry [10], and Horava-Lifshitz gravity [11]-[12].

The modification of the standard energy-momentum dispersion relation has motivated the development of double special relativity [13]. In this theory, apart from the velocity of light being the maximum velocity attainable, there is also a maximum energy scale in nature. This energy scale is the Planck energy $E_{P}$, and it is not possible to for a particle to attain energies beyond this energy. The double special relativity has been generalized to curved spacetime, and this doubly general theory of relativity is called gravity's rainbow [14]. In this theory, the geometry of spacetime depends on the energy of the test particle. So, particles of different energy see the geometry of spacetime differently. Hence, the geometry of spacetime is represented by a family of energy dependent metrics forming a rainbow of metrics. This is the reason the theory has been called gravity's rainbow. In order to construct this theory, the modified energy-momentum dispersion relation is written as

$$
E^{2} f^{2}\left(E / E_{P}\right)-p^{2} g^{2}\left(E / E_{P}\right)=m^{2}
$$

where $E_{P}$ is the Planck energy. The functions $f\left(E / E_{P}\right)$ and $g\left(E / E_{P}\right)$ are called rainbow functions, and they are required to satisfy

$$
\lim _{E / E_{P} \rightarrow 0} f\left(E / E_{P}\right)=1, \quad \lim _{E / E_{P} \rightarrow 0} g\left(E / E_{P}\right)=1 .
$$

This condition is needed, as the theory is constrained to reproduce the standard dispersion relation in the infrared limit. Motivated by this energy dependent modification to the dispersion relation, the metric $h(E)$ in gravity's rainbow is written as [15]

$$
h(E)=\eta^{a b} e_{a}(E) \otimes e_{b}(E) .
$$

Here the energy dependence of the frame fields can be written as

$$
e_{0}(E)=\frac{1}{f\left(E / E_{P}\right)} \tilde{e}_{0}, \quad e_{i}(E)=\frac{1}{g\left(E / E_{P}\right)} \tilde{e}_{i},
$$

\footnotetext{
* email address: hendi@shirazu.ac.ir

† email address: f2mir@uwaterloo.ca
} 
where the tilde quantities refer to the energy independent frame fields. The energy at which the spacetime is probed is represented by $E$, and the maximum attainable energy is represented by $E_{p}$. So, if a text particle is used to probe the geometry of spacetime, then $E$ is the energy of that test particle. Thus, by definition $E$ cannot become greater than $E_{p}[15]$. As we will be applying gravity's rainbow to the black hole thermodynamics, the energy $E$ will correspond to the energy a quantum particle in the vicinity of the event horizon, which is emitted in the Hawking radiation [16]-[20]. It is possible to translate the uncertainty principle $\Delta p \geq 1 / \Delta x$ into a bound on energy $E \geq 1 / \Delta x$ 21]. In gravity's rainbow, even though the metric depends on the energy, the usual uncertainty principle still holds [22]. It has been demonstrated that the uncertainty in the position of the particle near the horizon should be equal to the radius of the event horizon radius [16]-20]

$$
E \geq 1 / \Delta x \approx 1 / r_{+} .
$$

This energy $E$ of the particle in the vicinity of the event horizon will be used in the rainbow functions. It may be noted that this energy $E$ is a dynamical function of the radial coordinate [23]. We will not need the explicit dependence of this energy on the radial coordinate, but it is important to note that the rainbow functions are dynamical, and so they cannot be gauged away. Furthermore, this energy of a particle near the horizon is bounded by the Planck energy $E_{p}$, and it cannot increase to an arbitrary value.

This bound on the energy also modifies the temperature of the black, and this modification to the temperature of the black hole can be used to calculate the corrections to the entropy of the black hole in gravity's rainbow [16]. The modification of the thermodynamics of black rings in gravity's rainbow has also been calculated 24]. It has been observed that the temperature of both the black holes and black rings starts to reduce after attaining a maximum value. Thus, at a critical size the temperature of the black holes and black rings becomes zero. At this value the entropy also becomes zero. Thus, the back hole stops radiating Hawking radiation, after reaching this critical radius. Thus, the gravity's rainbow predicts the existence of a black remnant. It has been argued that such a remnant will form for all black objects 25]. In fact, it has been explicitly demonstrated that such a remnant does form for Kerr black holes, Kerr-Newman black holes in de Sitter space, charged AdS black holes, higher dimensional Kerr-AdS black holes and black saturn [25].

It may be noted that the behavior of gravity's rainbow depends on the form of the rainbow functions chosen. The construction of these rainbow functions is motivated from various theoretical and phenomenological considerations. Even though the form of these rainbow functions is different, the main property of these rainbow functions is that they make the geometry of spacetime dependent on the energy of the probe. This is the property which we will require in our analysis. So, in this paper, we will analyze the behavior of black hole solutions in the Gauss-Bonnet gravity's rainbow using these phenomenologically motivated rainbow functions.

\section{GAUSS-BONNET GRAVITY'S RAINBOW}

It may be noted that it is possible to add higher order curvature terms to the original Lagrangian for general relativity. The theory obtained by adding quadratic powers of the curvature terms is called Gauss-Bonnet gravity. It is also possible to construct a Gauss-Bonnet-Maxwell gravity [26]-[29]. The Lagrangian of Gauss-Bonnet-Maxwell gravity can be written as

$$
L_{\text {tot }}=R-2 \Lambda+\alpha\left(R_{a b c d} R^{a b c d}-4 R_{a b} R^{a b}+R^{2}\right)-\mathcal{F},
$$

where $R_{a b c d}$ and $\Lambda$ are the Riemann tensor and the cosmological constant, respectively. Here $\alpha$ denotes the GaussBonnet (GB) coefficient, $R=R_{a}^{a}$ denotes the Ricci scalar and $R_{c d}=R_{c a d}^{a}$ denotes the Ricci tensor. The last term in Eq. (66) is the Maxwell invariant $\mathcal{F}=F_{a b} F^{a b}$, where $F_{a b}=\partial_{a} A_{b}-\partial_{b} A_{a}$ is the electromagnetic field tensor and $A_{b}$ is the gauge potential. Now the fields equations for the the Gauss-Bonnet-Maxwell gravity Lagrangian (6) can be written as

$$
G_{a b}^{E}+\Lambda g_{a b}+\alpha G_{a b}^{G B}=-\frac{1}{2} g_{a b} \mathcal{F}+2 F_{a c} F_{b}^{c},
$$

where

$$
\nabla_{a} F^{a b}=0 .
$$

Here the the Einstein tensor is denoted by $G_{a b}^{E}$ and

$$
\begin{aligned}
G_{a b}^{G B}= & 2\left(R_{a c d e} R_{b}^{c d e}-2 R_{a c b d} R^{c d}-2 R_{a c} R_{b}^{c}+R R_{a b}\right)- \\
& \frac{1}{2}\left(R_{c d e f} R^{c d e f}-4 R_{c d} R^{c d}+R^{2}\right) g_{a b} .
\end{aligned}
$$


In gravity's rainbow the spacetime geometry depends on the energy of the probe $(E)$. We can follow the methods used in the usual gravity's rainbow [13], and absorb all the energy dependence of the Gauss-Bonnet gravity's rainbow into the rainbow functions. So, we can write the energy dependent metric Gauss-Bonnet gravity's rainbow as

$$
d \tau^{2}=-d s^{2}=\frac{\Psi(r)}{f(E)^{2}} d t^{2}-\frac{1}{g(E)^{2}}\left(\frac{d r^{2}}{\Psi(r)}+r^{2} d \Omega^{2}\right)
$$

where

$$
d \Omega^{2}=d \theta_{1}^{2}+\sum_{i=2}^{d-2} \prod_{j=1}^{i-1} \sin ^{2} \theta_{j} d \theta_{i}^{2}
$$

Since we are looking for the black hole solutions with a radial electric field, we know that the nonzero components of the electromagnetic field are

$$
F_{t r}=-F_{r t}
$$

One can use Eq. (8) with the metric (9) to obtain the following explicit form of $F_{t r}$

$$
F_{t r}=\frac{q}{r^{d-2}}
$$

where $q$ is an integration constant related to the electric charge of the black hole. In addition, it is easy to show that the following metric function satisfies all of the field equations (7), simultaneously

$$
\Psi(r)=1+\frac{r^{2}}{2 \alpha^{\prime} g(E)^{2}}(1-\sqrt{\Theta(r)})
$$

with

$$
\Theta(r)=1+\frac{8 \alpha^{\prime}}{(d-1)(d-2)}\left(\Lambda+\frac{(d-1)(d-2) m}{2 r^{d-1}}-\frac{(d-1)(d-3) q^{2} f(E)^{2} g(E)^{2}}{r^{2 d-4}}\right)
$$

where $m$ is an integration constant that is related to mass and $\alpha^{\prime}=(d-3)(d-4) \alpha$. Taking into account Eqs. (12) and (13), it is clear that one cannot remove rainbow functions with rescaling.

The choice of the rainbow functions $f\left(E / E_{p}\right)$ and $g\left(E / E_{p}\right)$ is very important for making predictions. That choice is preferred to be based on phenomenological motivations. Many proposals exist in the literature, we will some forms which have important phenomenological motivations.

The rainbow functions motivated by the results obtained from loop quantum gravity and non-commutative geometry are given by [30]-[31]

$$
f\left(E / E_{p}\right)=1, \quad g\left(E / E_{p}\right)=\sqrt{1-\eta\left(E / E_{p}\right)^{n}}
$$

Now using these rainbow functions the metric is given by

$$
\Psi_{1}(r)=1+\frac{r^{2}}{2 \alpha^{\prime}\left[1-\eta\left(\frac{E}{E_{p}}\right)^{n}\right]}\left(1-\sqrt{\Theta_{1}(r)}\right)
$$

with

$$
\Theta_{1}(r)=1+\frac{8 \alpha^{\prime}}{(d-1)(d-2)}\left(\Lambda+\frac{(d-1)(d-2) m}{2 r^{d-1}}-\frac{(d-1)(d-3) q^{2}\left[1-\eta\left(\frac{E}{E_{p}}\right)^{n}\right]}{r^{2 d-4}}\right)
$$

The hard spectra from gamma-ray burster's are given by can also be used to motivate the construction of rainbow functions [32]. These rainbow functions are given by

$$
f\left(E / E_{p}\right)=\frac{e^{\beta E / E p}-1}{\beta E / E_{p}}, \quad g\left(E / E_{p}\right)=1 .
$$


The metric corresponding to Eqs. (13), (14) and (18), can be written as

$$
\Psi_{2}(r)=1+\frac{r^{2}}{2 \alpha^{\prime}}\left(1-\sqrt{\Theta_{2}(r)}\right),
$$

with

$$
\Theta_{2}(r)=1+\frac{8 \alpha^{\prime}}{(d-1)(d-2)}\left(\Lambda+\frac{(d-1)(d-2) m}{2 r^{d-1}}-\frac{(d-1)(d-3) q^{2} E_{p}^{2}\left(e^{\frac{\beta E}{E p}}-1\right)^{2}}{\beta^{2} E^{2} r^{2 d-4}}\right)
$$

rainbow functions in which the velocity of light is constant have also been analyzed. This choice of rainbow functions is given by 33 .

$$
f\left(E / E_{p}\right)=g\left(E / E_{p}\right)=\frac{1}{1-\lambda E / E_{p}} .
$$

The metric function corresponding to the case where the velocity of light is a constant can be written as (18)

$$
\Psi_{3}(r)=1+\frac{r^{2}\left(1-\frac{\lambda E}{E_{p}}\right)^{2}}{2 \alpha^{\prime}}\left(1-\sqrt{\Theta_{3}(r)}\right),
$$

with

$$
\Theta_{3}(r)=1+\frac{8 \alpha^{\prime}}{(d-1)(d-2)}\left(\Lambda+\frac{(d-1)(d-2) m}{2 r^{d-1}}-\frac{(d-1)(d-3) q^{2}}{r^{2 d-4}\left(1-\frac{\lambda E}{E_{p}}\right)^{4}}\right) .
$$

It may be noted that the bounds on the values of $\beta, \eta, \lambda$ has been analyzed using various theoretical and experimental considerations 34 .

\section{THERMODYNAMICS AND THERMAL STABILITY}

In this section, we will analyze the thermodynamics of the black hole solution obtained in the previous section. It is possible to obtain the Hawking temperature by using the surface gravity,

$$
T=\frac{g(E)}{r_{+} f(E)} \frac{(d-2)(d-3)\left(1+\frac{\alpha^{\prime}(d-5) g^{2}(E)}{(d-3) r_{+}^{2}}\right)-\frac{2 \Lambda r_{+}^{2}}{g^{2}(E)}-\frac{2 f^{2}(E)(d-3)^{2} q^{2}}{r_{+}^{2 d-6}}}{4 \pi(d-2)\left(1+\frac{2 \alpha^{\prime} g^{2}(E)}{r_{+}^{2}}\right)} .
$$

According to the area law, the entropy of black holes is equal to one-quarter of the horizon area. This relation is valid for Einstein gravity, whereas we are not allowed to use it for higher derivative gravity. In this paper we investigate it in Gauss-Bonnet gravity's rainbow. We can use the Wald formula for calculating the entropy

$$
S=\frac{1}{4} \int d^{n-1} x \sqrt{\gamma}(1+2 \alpha \widetilde{R})
$$

where $\widetilde{R}$ is the Ricci scalar for the induced metric $\gamma_{a b}$ on the $(d-2)$ dimensional boundary. We obtain

$$
S=\frac{V_{d-2} r_{+}^{d-2}}{4 g^{d-2}(E)}\left(1+\frac{2(d-2) \alpha^{\prime} g^{2}(E)}{(d-4) r_{+}^{2}}\right),
$$

which confirms that the obtained black hole solutions violate the area law. We should mention that $V_{d-2}$ denotes the volume of $(d-2)$-dimensional sphere.

Considering the Gauss law and calculating the flux of the electric field at infinity, one can find the electric charge of the black hole has the following form

$$
Q=\frac{V_{d-2}(d-3) q}{4 \pi} \frac{f(E)}{g^{d-3}(E)}
$$


We can calculate the electric potential $\Phi$ as

$$
\Phi=\left.A_{\mu} \chi^{\mu}\right|_{r \longrightarrow \infty}-\left.A_{\mu} \chi^{\mu}\right|_{r \longrightarrow r_{+}}=\frac{q}{r_{+}^{d-3}},
$$

where $\chi^{\mu}$ is the null generator of the horizon.

In order to calculate the finite mass of the black hole we will use the ADM (Arnowitt-Deser-Misner) approach for large values of $r$

$$
M=\frac{V_{d-2}}{16 \pi} \frac{m(d-2)}{f(E) g^{d-1}(E)} .
$$

Now using Eqs. (13), (26) and (27) and considering $M$ as a function of the extensive parameters $S$ and $Q$, we obtain the following result,

$$
\begin{aligned}
M(S, Q)= & \frac{(d-2)}{16 \pi f(E) g^{d-1}(E)}\left[g^{2}(E) r_{+}^{d-3}\left(1+\frac{\alpha^{\prime} g^{2}(E)}{r_{+}^{2}}\right)\right. \\
& \left.-\frac{2 \Lambda r_{+}^{d-1}}{(d-1)(d-2)}+\frac{32 \pi^{2} Q^{2} g^{2 d-4}(E)}{(d-2)(d-3) r_{+}^{d-3}}\right]
\end{aligned}
$$

We can calculate the temperature and electric potential as the intensive parameter using the following relation

$$
T=\left(\frac{\partial M}{\partial S}\right)_{Q}=\left(\frac{\partial M}{\partial r_{+}}\right)_{Q} /\left(\frac{\partial S}{\partial r_{+}}\right)_{Q} \quad, \Phi=\left(\frac{\partial M}{\partial Q}\right)_{S}
$$

It may be noted that these relations are similar to the relations obtained in Eqs. (24) and (28, Thus, the conserved and thermodynamic quantities satisfy the first law of thermodynamics,

$$
d M=T d S+\Phi d Q
$$

It may be noted that we can investigate the thermal stability of the charged black hole solutions of Gauss-Bonnet gravity's rainbow through the canonical ensemble. In this ensemble, the electric charge is set as a fixed parameter and, therefore, the positivity of the heat capacity

$$
C_{Q}=\frac{T_{+}}{\left(\frac{\partial^{2} M}{\partial S^{2}}\right)_{Q}}
$$

is sufficient to ensure the local stability. Since we are investigating the physical black hole solutions with positive temperature, it is sufficient to examine the positivity of

$$
\left(\frac{\partial^{2} M}{\partial S^{2}}\right)_{Q}=-\frac{(d-2) r_{+}^{d-4}\left[(d-4) \alpha g^{2}(E)+\frac{r_{+}^{2}}{2(d-3)}\right]^{2} \Upsilon_{1}}{2 g^{d-2}(E) \Upsilon_{2}}
$$

where

$$
\begin{aligned}
\Upsilon_{1}= & -\frac{2(d-3) q^{2} f^{2}(E) g^{2}(E)}{(d-2) r_{+}^{2 d-8}}+(d-4)(d-5) \alpha g^{4}(E)+g^{2}(E) r_{+}^{2} \\
& -\frac{2 \Lambda r_{+}^{4}}{(d-2)(d-3)}, \\
\Upsilon_{2}= & -\frac{2(d-3) q^{2} f^{2}(E) g^{2}(E)\left[(d-4)(2 d-7) \alpha g^{2}(E)+\frac{(2 d-5) r_{+}^{2}}{2(d-3)}\right]}{(d-2) r_{+}^{2 d-8}}+ \\
& (d-4)^{2}(d-5) \alpha^{2} g^{6}(E)+\frac{(d-4)(d-9) \alpha g^{4}(E) r_{+}^{2}}{2(d-3)}+ \\
& \frac{g^{2}(E)[d-2+12 \alpha \Lambda(d-4)] r_{+}^{4}}{2(d-2)(d-3)}+\frac{\Lambda r_{+}^{6}}{(d-2)(d-3)^{2}}
\end{aligned}
$$



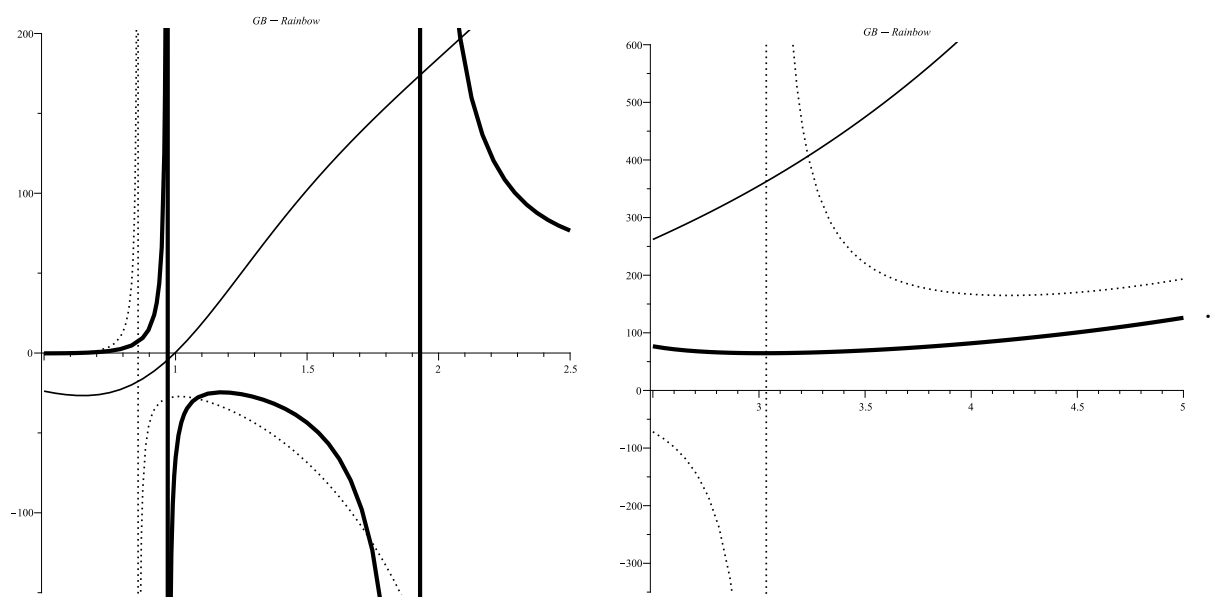

FIG. 1: Model 1: $C_{Q}$ versus $r_{+}$for $E=1, E_{p}=5, \Lambda=-1, q=1, \eta=1, n=2, \alpha=5$, and $d=5$ (solid line), $d=6$ (bold line) and $d=7$ (dotted line). "different scales"
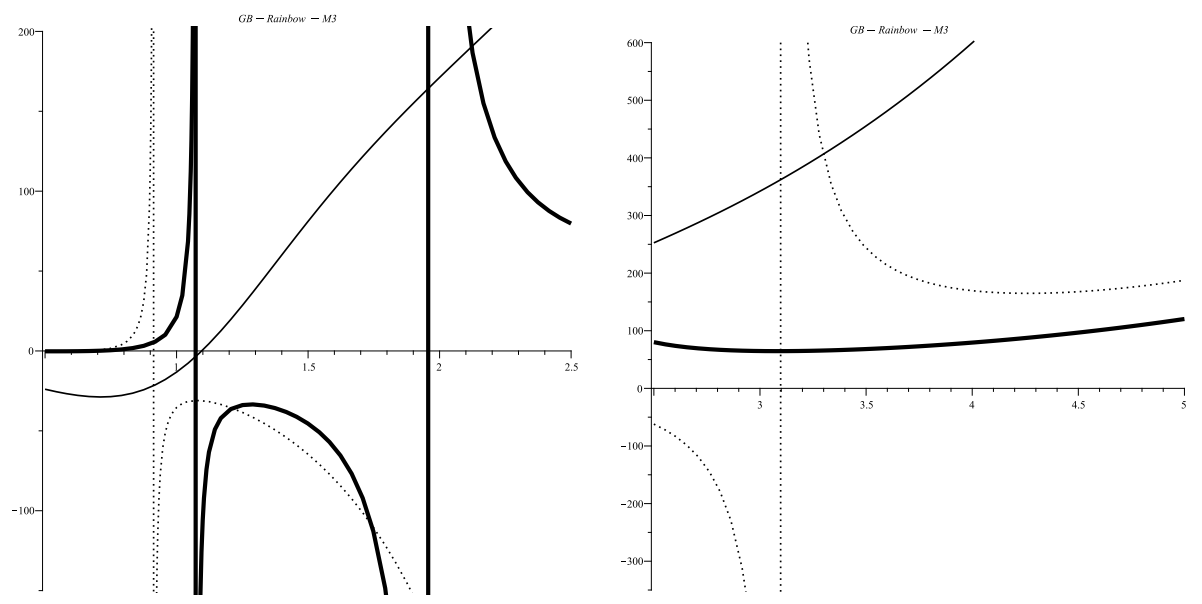

FIG. 2: Model 2: $C_{Q}$ versus $r_{+}$for $E=1, E_{p}=5, \Lambda=-1, q=1, \beta=2, \alpha=5$, and $d=5$ (solid line), $d=6$ (bold line) and $d=7$ (dotted line). "different scales"
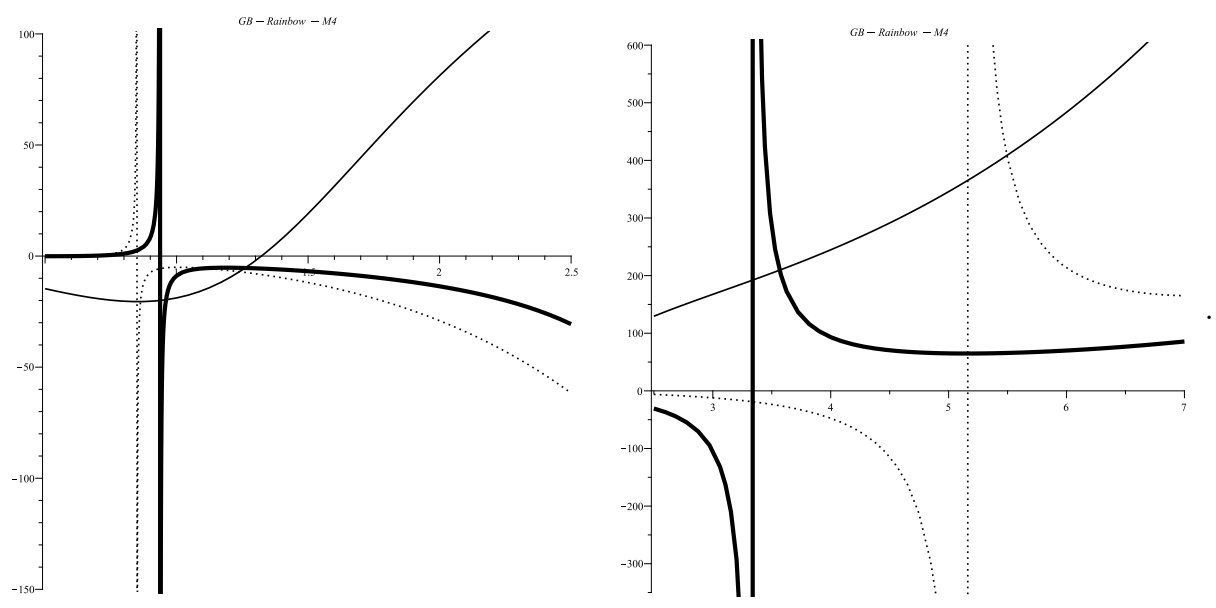

FIG. 3: Model 3: $C_{Q}$ versus $r_{+}$for $E=1, E_{p}=5, \Lambda=-1, q=1, \lambda=2, \alpha=5$, and $d=5$ (solid line), $d=6$ (bold line) and $d=7$ (dotted line). "different scales" 

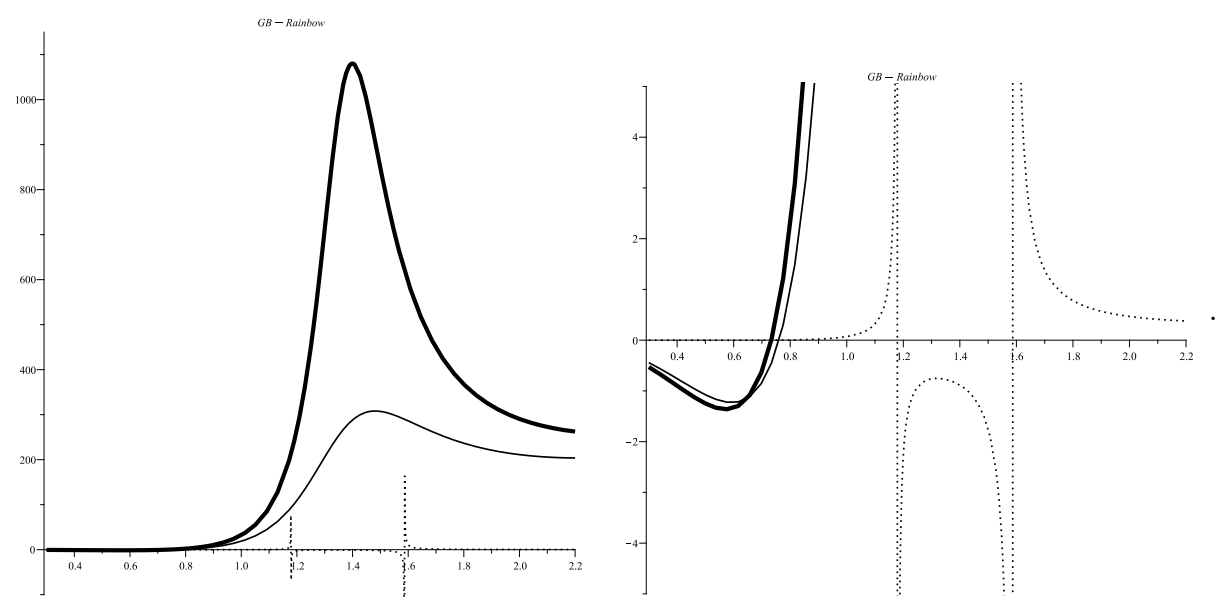

FIG. 4: Model 1: $C_{Q}$ versus $r_{+}$for $E=1, E_{p}=5, \Lambda=-1, q=1, \eta=1, n=2, d=6$, and $\alpha=2$ (solid line), $\alpha=2.4$ (bold line) and $\alpha=3$ (dotted line). "different scales"
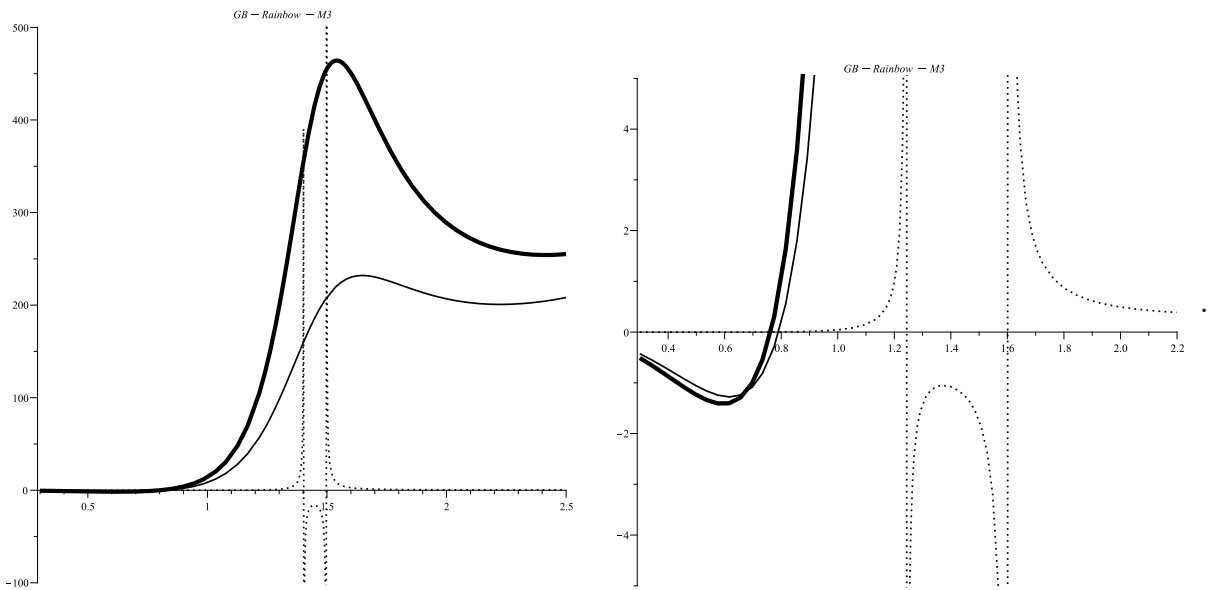

FIG. 5: Model 2: $C_{Q}$ versus $r_{+}$for $E=1, E_{p}=5, \Lambda=-1, q=1, \beta=2, d=6$, and $\alpha=2$ (solid line), $\alpha=2.4$ (bold line) and $\alpha=3$ (dotted line). "different scales"
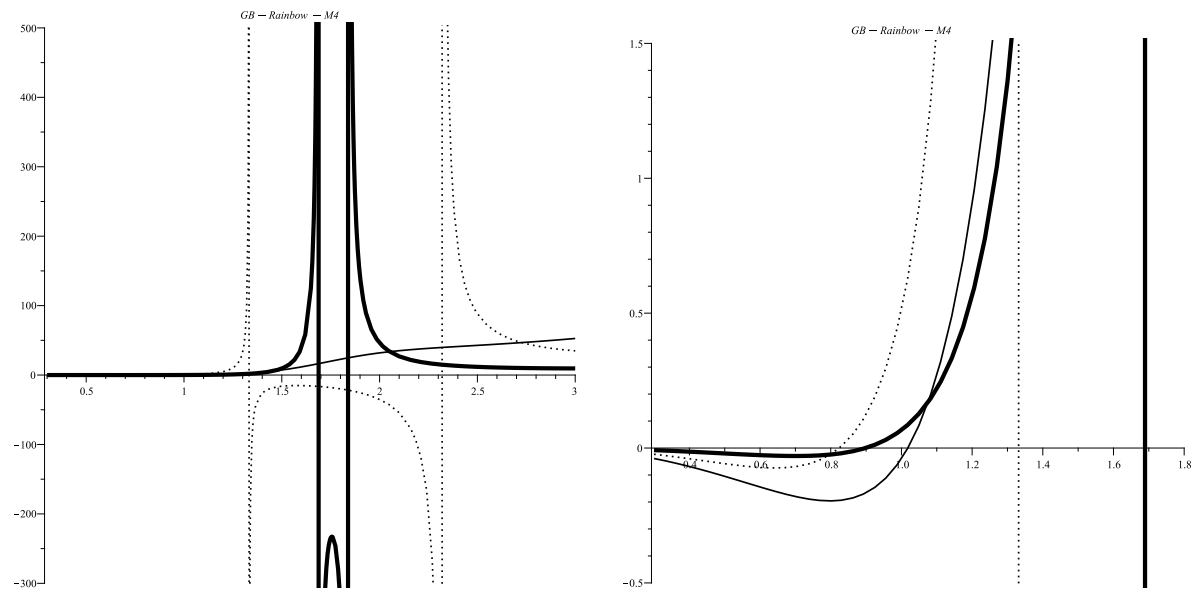

FIG. 6: Model 3: $C_{Q}$ versus $r_{+}$for $E=1, E_{p}=5, \Lambda=-1, q=1, \lambda=2, d=6$, and $\alpha=0.5$ (solid line), $\alpha=1$ (bold line) and $\alpha=1.5$ (dotted line). "different scales" 

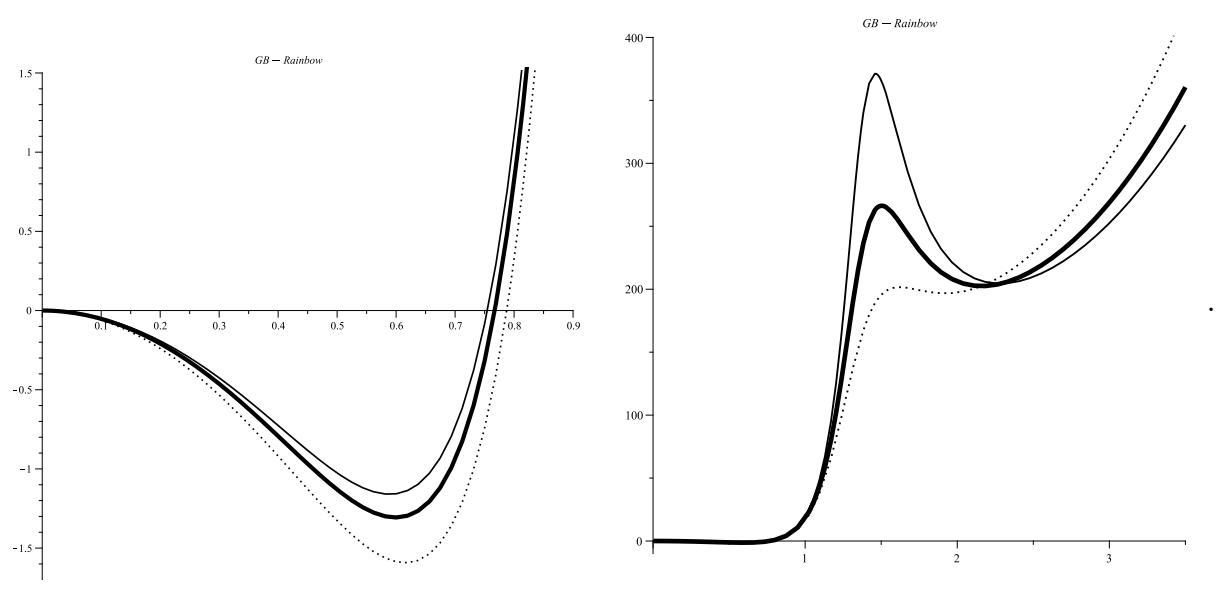

FIG. 7: Model 1: $C_{Q}$ versus $r_{+}$for $E=1, E_{p}=5, \Lambda=-1, q=1, \alpha=2, n=2, d=6$, and $\eta=0$ (solid line), $\eta=2$ (bold line) and $\eta=5$ (dotted line). "different scales"
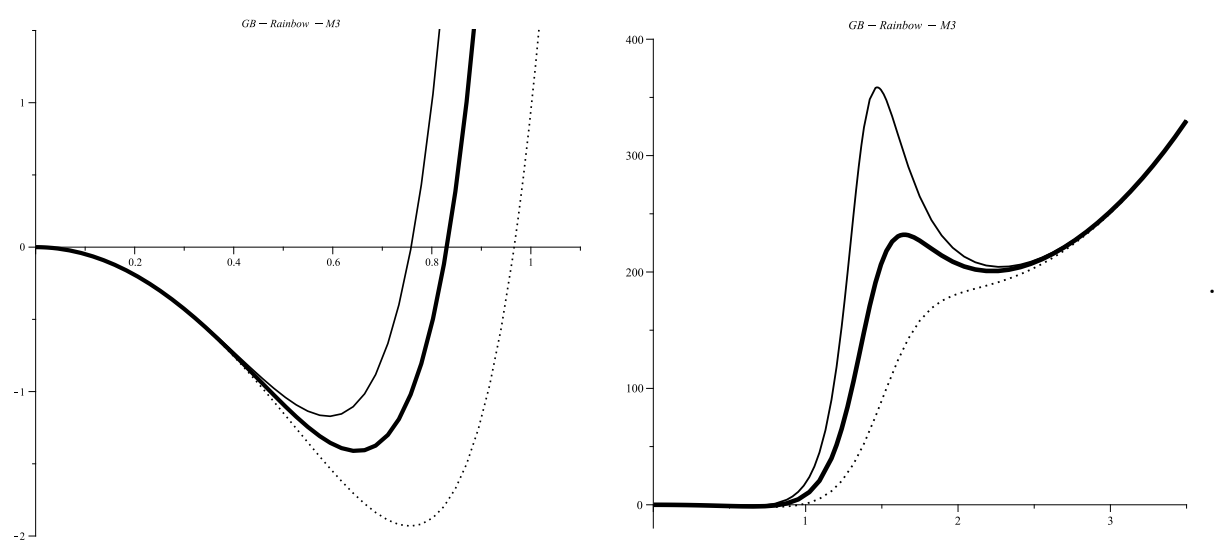

FIG. 8: Model 2: $C_{Q}$ versus $r_{+}$for $E=1, E_{p}=5, \Lambda=-1, q=1, \alpha=2, d=6$, and $\beta=0.1$ (solid line), $\beta=2$ (bold line) and $\beta=5$ (dotted line). "different scales"

Thus, we are able to derive an explicit expression for the energy dependence of the heat capacity of a black hole in Gauss-Bonnet gravity's rainbow. The positivity of this heat capacity ensures the local stability.

In order to discuss the effects of gravity's rainbow on thermal stability, we will analyze various different choices of rainbow functions. We plot heat capacity versus horizon radius for these different choices of rainbow functions. We also investigate the effects of $d$ (spacetime dimensions), $\alpha$ (GB parameter) and the rainbow parameters $(\eta, n, \beta, \lambda$ on the heat capacity.

The head capacity also depends on the dimensions of spacetime, as can be observed from Figs. 1]3, These figures demonstrate that divergent points appear in higher dimensions. Depending on the values of the parameters, one may find that for low dimensional cases, $C_{Q}$ is a smooth function and with a real positive root. However, by increasing the dimensions of spacetime, divergences can occur for $C_{Q}$. In fact, two divergences occur and $C_{Q}$ is negative in between these two divergent points. This makes the system unstable. Although the global behavior of $C_{Q}$ is uniform for different choices of rainbow functions, their local properties such as slopes and the location of the root, along with the occurrence of divergences are different for different choices of rainbow functions.

All figures indicate that $C_{Q}$ has a real positive root at $r_{+0}$ for all values of parameters. The location of $r_{+0}$ will be affected by variation of the parameters. It can be observed from Figs. 1,13, that by increasing $d, \alpha$ and $n$ the value of $r_{+0}$ decreases. Furthermore, by increasing $\eta, \beta$ and $\lambda$ the value of $r_{+0}$ increases. It is possible to choose special values of the parameters such that the heat capacity does not diverge and remains a smooth function. However, it is also possible to fix the free parameters in such a way that $C_{Q}$ has two real positive roots at $r_{+1}$ and $r_{+2}\left(r_{+1}<r_{+2}\right)$. In Figs. 113, we find that there is a critical value for the GB parameter $\left(\alpha_{c}\right)$, such that $\alpha>\alpha_{c}$, and for this value of $\alpha$, the heat capacity has two divergent points. Furthermore, $C_{Q}$ is a regular function for $\alpha<\alpha_{c}$. It may be noted that the value of $\alpha_{c}$ depends on the values of other parameters. In addition, considering the case of having two divergent 

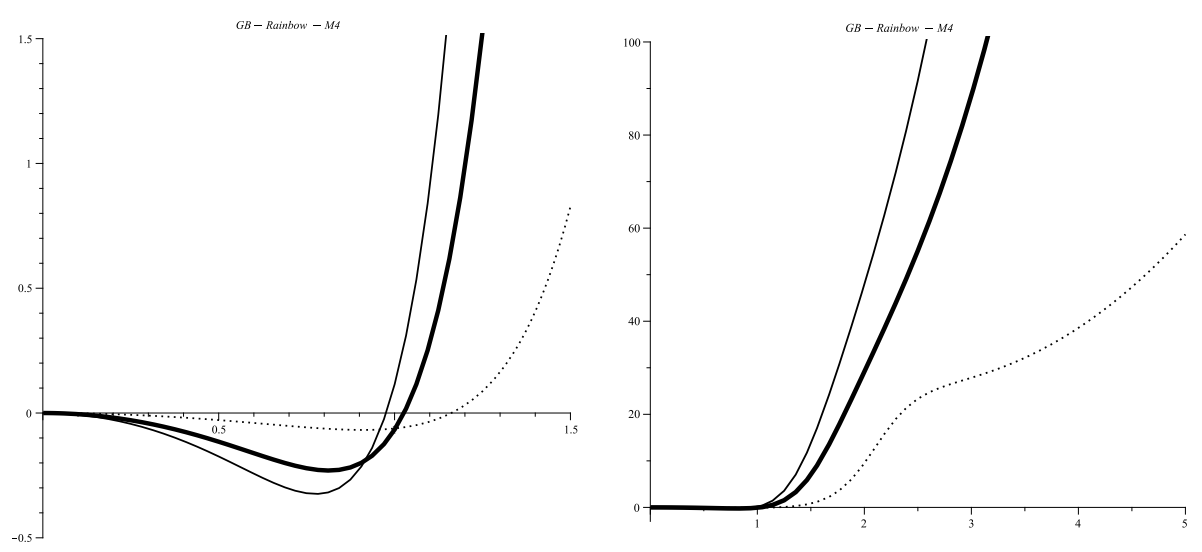

FIG. 9: Model 3: $C_{Q}$ versus $r_{+}$for $E=1, E_{p}=5, \Lambda=-1, q=1, \alpha=0.3, d=6$, and $\lambda=0.1$ (solid line), $\lambda=1$ (bold line) and $\lambda=3$ (dotted line). "different scales"
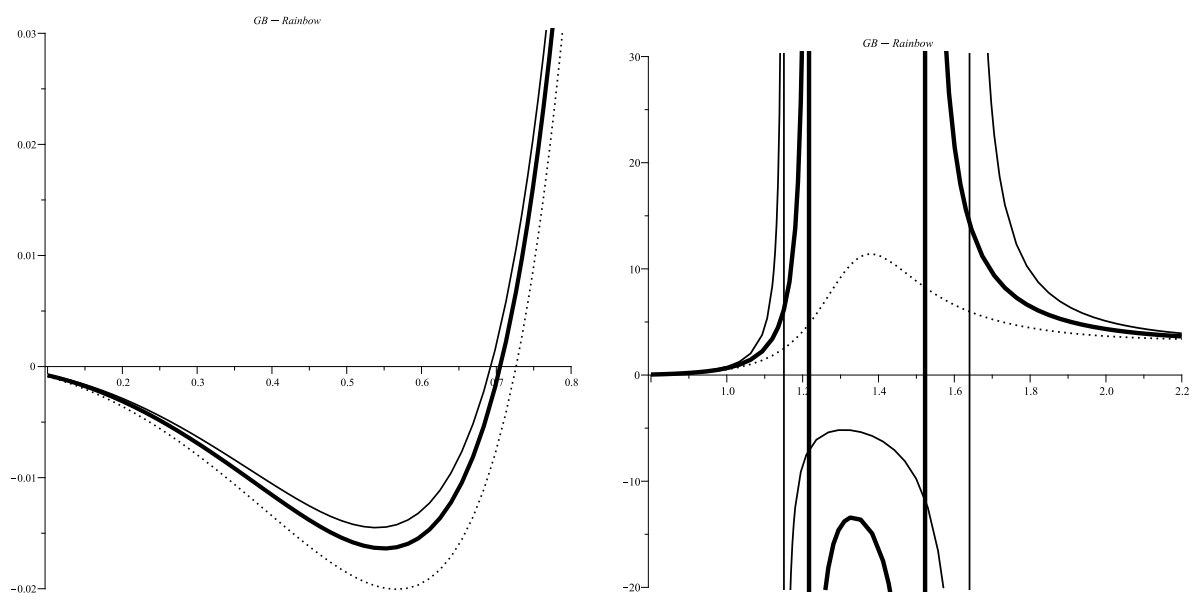

FIG. 10: Model 1: $C_{Q}$ versus $r_{+}$for $E=1, E_{p}=5, \Lambda=-1, q=1, \alpha=3, n=2, d=6$, and $\eta=0$ (solid line), $\eta=2$ (bold line) and $\eta=5$ (dotted line). "different scales"
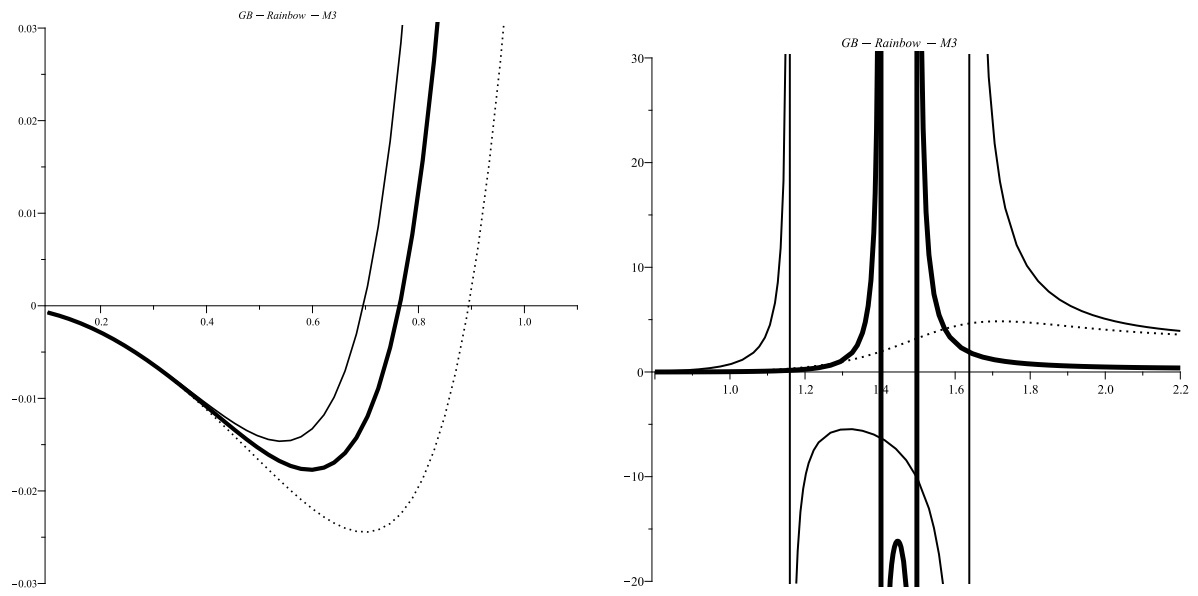

FIG. 11: Model 2: $C_{Q}$ versus $r_{+}$for $E=1, E_{p}=5, \Lambda=-1, q=1, \alpha=3, d=6$, and $\beta=0.1$ (solid line), $\beta=2$ (bold line) and $\beta=5$ (dotted line). "different scales" 

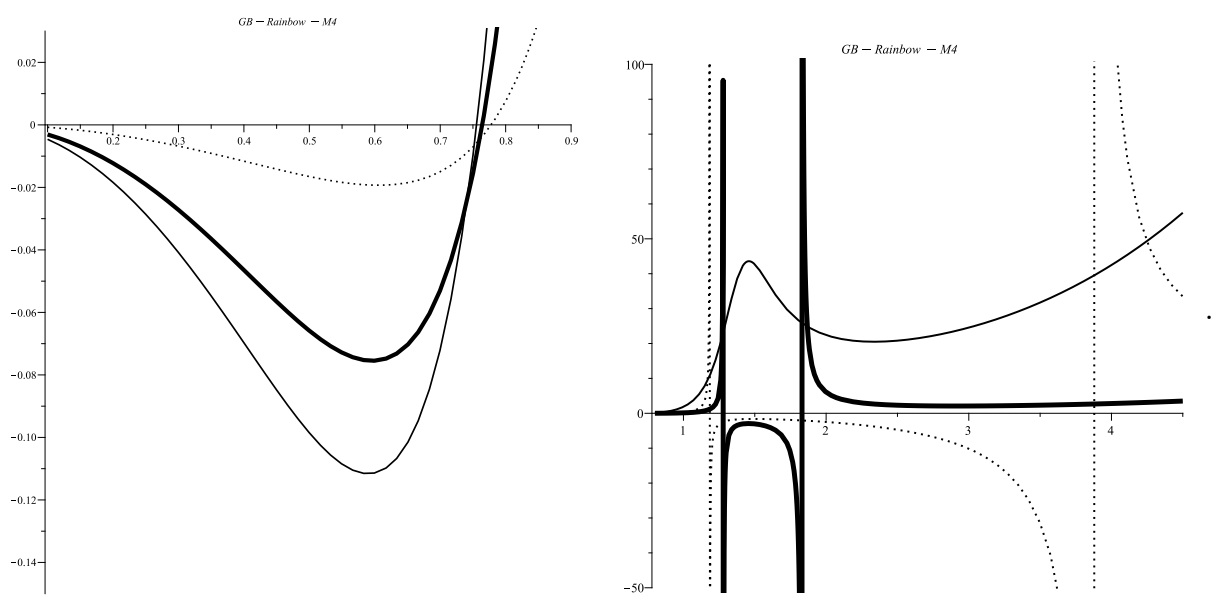

FIG. 12: Model 3: $C_{Q}$ versus $r_{+}$for $E=1, E_{p}=5, \Lambda=-1, q=1, \alpha=2, d=6$, and $\lambda=0.1$ (solid line), $\lambda=1$ (bold line) and $\lambda=3$ (dotted line). "different scales"
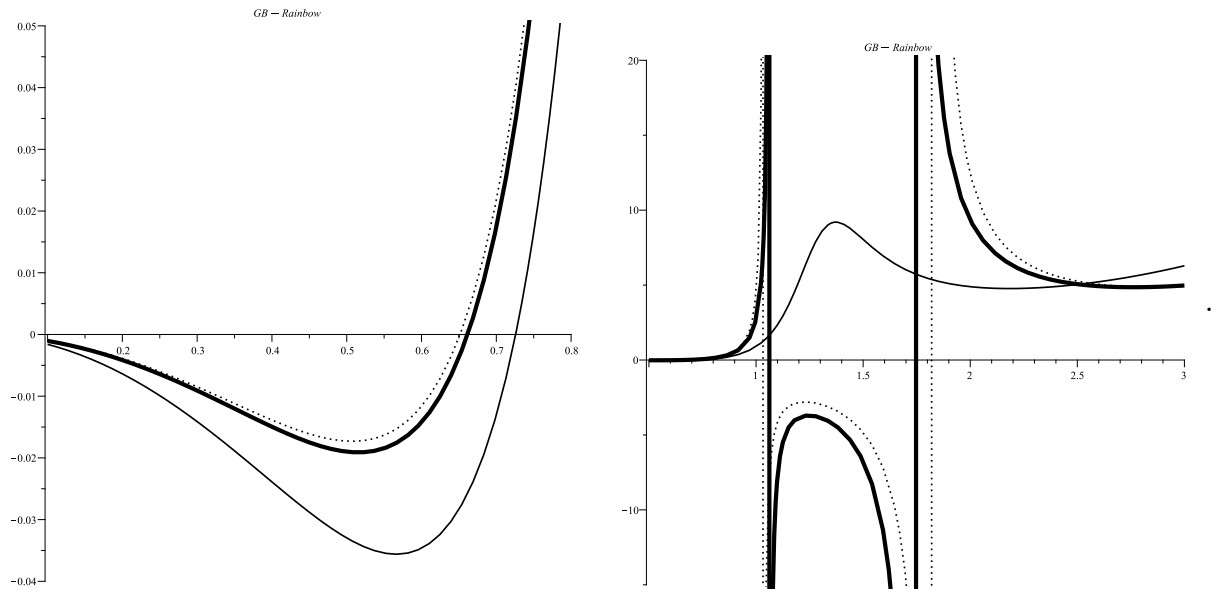

FIG. 13: Model 1: $C_{Q}$ versus $r_{+}$for $E=1, E_{p}=5, \Lambda=-1, q=1, \alpha=4, \eta=2, d=6$, and $n=1$ (solid line), $n=2$ (bold line) and $n=3$ (dotted line). "different scales"

points in Fig. 10, we find that one can obtain a smooth $C_{Q}$ by increasing the value of $\eta$ and $\beta$ (decreasing $\lambda$ ). The same behavior can be obtained by decreasing the value of $n$. In other words, since we regard $0<\frac{E}{E_{p}}<1$, one finds that the effects of varying $\eta$ and $\beta$ are opposite to the effects of varying $n$ and $\lambda$. Thus, the effect of gravity's rainbow decreases by that decreasing $\eta, \beta$ and increasing $n, \lambda$.

\section{CONCLUSION}

In this paper, motivated by the developments in gravity's rainbow, we have developed a generalization of GaussBonnet gravity to an energy dependent version of this theory. This new theory called Gauss-Bonnet gravity's rainbow has been used for analyzing black hole solutions. In this theory, the metric in the Gauss-Bonnet gravity is made energy dependent in such a way that the regular Gauss-Bonnet gravity is recovered in the infrared limit. We have explicitly calculated the modifications to the thermodynamics of black holes in Gauss-Bonnet's gravity's rainbow. This was done by using various phenomenologically motivated rainbow functions. We used the rainbow functions whose construction was motivated by hard spectra of gamma-ray bursters at cosmological distances [30]. We also used the rainbow functions whose construction was motivated from results that have been obtained in loop quantum gravity and non-commutative geometry [32]. Finally, as it is possible to construct the rainbow functions in which the velocity of light is constant [33], we also used these to analyze our results corresponding to such rainbow functions. We have also demonstrated that the first law of thermodynamics still holds for this modified thermodynamics. Finally, we 
commented about the thermodynamical stability of these solutions. We showed that $C_{Q}$ has two different behaviors. In the first case it is a smooth and regular function with a real positive root at $r_{+0}$. In this case black hole solutions are thermally stable for $r_{+}>r_{+0}$. For the second case, there are two singular (divergence) points for the heat capacity in which they are corresponding to second order phase transition points. We showed that variation of the model parameters, dimensionality and GB parameter can affect on the heat capacity behavior.

It may be noted that in higher dimensions it is possible to construct various interesting solutions to black holes like black rings and black saturns. The black rings [24], and black saturns [25] have been analyzed using gravity's rainbow. It has been demonstrated that in gravity's rainbow these solutions also have a remnant. It will be interesting to construct black rings and black saturn solutions in Gauss-Bonnet gravity's rainbow. It is expected that these solutions will also have a remnant because of the modification of the thermodynamics due to the rainbow functions. It has also been recently argued that the energy needed to create a mini black hole in a particle accelerator will increase in gravity's rainbow. Thus, it would be possible to test a certain limit of gravity's rainbow at the LHC [35]. This increase in energy occurs as the energy needed to form a mini black hole at the LHC has to be greater than the energy of the black hole remnant. It will be interesting to analyze a similar situation using Gauss-Bonnet gravity's rainbow. It has been proposed that gravity's rainbow can be used to address the black hole information paradox [36]-[37]. So, it would also be interesting to repeat such an analysis for Gauss-Bonnet gravity's rainbow.

\section{Acknowledgments}

S. H. H. thanks the Shiraz University Research Council. The work of S. H. H has been supported financially by the Research Institute for Astronomy and Astrophysics of Maragha, Iran.

[1] R. Iengo, J. G. Russo and M. Serone, JHEP 0911 (2009) 020

[2] A. Adams, N. Arkani-Hamed, S. Dubovsky, A. Nicolis and R. Rattazzi, JHEP 0610 (2006) 014

[3] B. M. Gripaios, JHEP 0410 (2004) 069

[4] J. Alfaro, P. Gonzalez and R. Avila, Phys. Rev. D 91 (2015) 105007

[5] H. Belich and K. Bakke, Phys. Rev. D 90 (2014) 025026

[6] G. 't Hooft, Class. Quant. Grav. 13 (1996) 1023

[7] V. A. Kostelecky and S. Samuel, Phys. Rev. D 39 (1989) 683.

[8] G. Amelino-Camelia, J. R. Ellis, N. Mavromatos, D. V. Nanopoulos, and S. Sarkar, Nature 393 (1998) 763

[9] R. Gambini and J. Pullin, Phys. Rev. D 59 (1999) 124021

[10] S. M. Carroll, J. A. Harvey, V. A. Kostelecky, C. D. Lane, and T. Okamoto, Phys. Rev. Lett. 87 (2001) 141601,

[11] P. Horava, Phys. Rev. D 79 (2009) 084008

[12] P. Horava, Phys. Rev. Lett. 102 (2009) 161301,

[13] J. Magueijo, and L. Smolin, Phys. Rev. D 71, 026010 (2005 )

[14] J. Magueijo and L. Smolin, Class. Quant. Grav. 21 (2004) 1725

[15] J.-J. Peng and S.-Q. Wu, Gen. Rel. Grav. 40 (2008) 2619

[16] A. F. Ali, Phys. Rev. D 89 (2014) 094021

[17] R. J. Adler, P. Chen and D. I. Santiago, Gen. Rel. Grav. 33 (2001) 2101

[18] M. Cavaglia, S. Das and R. Maartens, Class. Quant. Grav. 20 (2003) L205

[19] M. Cavaglia and S. Das, Class. Quant. Grav. 21 (2004) 4511

[20] G. Amelino-Camelia, M. Arzano and A. Procaccini, Phys. Rev. D 70 (2004) 107501

[21] E. M. Lifshitz, L. P. Pitaevskii and V. B. Berestetskii, Landau-Lifshitz Course of Theoretical Physics, Volume 4: Quantum Electrodynamics, Reed Educational and Professional Publishing, (1982)

[22] Y. Ling, X. Li, and H.-b. Zhang, Mod. Phys. Lett. A 22 (2007) 2749

[23] R. Garattini and E. N. Saridakis, arXiv:1411.7257 (2014)

[24] A. F. Ali, M. Faizal, and M. M. Khalil, JHEP 1412 (2014) 159

[25] A. F. Ali, M. Faizal, and M. M. Khalil, Nucl. Phys. B 894 (2015) 341

[26] M. H. Dehghani, S. H. Hendi, Phys. Rev. D 73, (2006) 084021

[27] S. H. Hendi, Phys. Lett. B 677, (2009) 123

[28] S. H. Hendi, S. Panahiyan and E. Mahmoudi, Eur. Phys. J. C 74 (2014) 3079

[29] S. H. Hendi and S. Panahiyan, Phys. Rev. D 90 (2014) 124008

[30] G. Amelino-Camelia, Living Reviews in Relativity 16 (2013) 5

[31] U. Jacob, F. Mercati, G. Amelino-Camelia and T. Piran, Phys. Rev. D 82 (2010) 084021

[32] G. Amelino-Camelia, J. R. Ellis, N. Mavromatos, D. V. Nanopoulos and S. Sarkar, Nature 393 (1998) 763

[33] J. Magueijo and L. Smolin, Phys. Rev. Lett. 190403 (2002) 88

[34] A. F. Ali, and M. M. Khalil, Europhys. Lett. 110 (2015) 20009 
[35] A. F. Ali, M. Faizal, and M. M. Khalil, Phys. Lett. B 743 (2015) 295

[36] A. F. Ali, M. Faizal, and B. Majumder, Europhys. Lett. 109 (2015) 20001

[37] Y. Gim and W. Kim, JCAP, 05 (2015) 002 